\title{
Facebook e pandemia da COVID-19: Um estudo sobre o compartilhamento de crenças online
}

\author{
Sheyla Fernandes ${ }^{1}$, Marcikele Nascimento ${ }^{1}$,Jennyfer Chagas ${ }^{1}$, \\ Jasielle Angelo ${ }^{1}$,Anna Rocha ${ }^{1}$ e Vagner Sousa ${ }^{2}$
}

${ }^{1}$ Universidade Federal de Alagoas, Brasil |

sheyla.fernandes@ip.ufal.br;nascimentomarcikele@gmail.com;jennymanda.ja@gmail.com; jassielle.angelo@gmail.com;annarochac140@gmail.com /https://orcid.org/0000-0003-4759-

1314; https://orcid.org/0000-0002-4844-743X; https://orcid.org/0000-0003-3077-9871; https://orcid.org/0000-0000-0000-0000; https://orcid.org/0000-0001-7849-589X

2Universidade de São Paulo, Brasil |vagner_souza@usp.br | https://orcid.org/0000-00000000-0000

Resumo: Introdução: A Síndrome Respiratória Aguda Grave por Coronavírus 2 (SARS-CoV2), apresentou-se como uma doença de alto potencial de contágio e que rapidamente se tornou uma emergência de saúde pública, sendo necessária a adoção de medidas sanitárias com a finalidade de conter seu avanço. As mudanças e incertezas comuns a esse período de crise promoveram impactos severos à saúde emocional da população. Com isso, as Redes Sociais Online (RSO) se tornaram facilitadoras da comunicação e compartilhamento de informações, promovendo uma rede de apoio, externalização das preocupações e estratégias de enfrentamento. Objetivos: Objetivou-se analisar a manifestação de crenças sobre a pandemia da COVID-19 entre usuários do Facebook no país. Métodos: Este estudo obteve seus dados através da busca com os descritores "COVID-19", "coronavírus" e "quarentena" em páginas e grupos abertos, que abordassem os assuntos em questão e apresentassem um número mínimo de $50 \mathrm{mil}$ curtidas/membros/seguidores no site Facebook (http://facebook.com.br). Os comentários foram coletados e transformados em dois corpus textuais analisados a partir do software Iramuteq pelo método da análise de similitude, tornando-se possível verificar a conexidade entre as palavras presentes. Resultados: Com a análise de similitude o primeiro corpus denominado "Humor" apresentou os termos: pai, amigo e quarentena como palavras centrais e em conexão. O segundo corpus denominado "Informação" apresentou os vocábulos centrais: deus, filho e pessoa. Conclusão: Através da análise das manifestações de crenças nas redes de apoio online diante da pandemia da COVID-19 no Brasil, foi possível traçar um olhar à luz do comportamento social diante de um período de crise sanitária de proporção global.

Palavras-chave: Covid-19; Quarentena; Coronavírus; Facebook.

Analysis of the Beliefs Manifested by Individuals on Facebook in Face of COVID-19

Abstract: Introduction: Severe Acute Respiratory Syndrome Coronavirus 2 (SARS-CoV-2), presented itself as a disease with high potential for contagion and that quickly became a public health emergency, requiring the adoption of some circulation and sanitary measures in order to contain the progress of the disease. These changes and uncertainties of the disease promote great psychological pressure, which can trigger psychological problems. With this, Online Social Networks (RSO) proved to be facilitators of communication and information sharing, promoting a support network, externalizing the beliefs of individuals and coping strategies. Objectives: The objective was to analyze the manifestation of beliefs about the pandemic of COVID-19 among Facebook users in the country. Methods: This study obtained its data through the search with the descriptors "COVID-19", "coronavirus" and "quarantine" in open pages and groups, which addressed the issues in question and presented a minimum number of 50 thousand likes / members / followers on the Facebook site (http://facebook.com.br). The comments were collected and transformed into two textual corpus analyzed using the Iramuteq software using the similarity analysis method, making it possible to verify the connection between the words present. Results: With the similitude analysis, the first corpus called "Humor" presented the terms: father, friend and quarantine as central and connected words. The second corpus called "Information" presented the central words: god, son and person. Conclusion: Through this analysis of the manifestations of beliefs in Online Social Networks in face of the pandemic of COVID-19 in Brazil, it was possible to draw a look in the light of social behavior in the face of a period of global health crisis.

Keywords: Covid-19; Quarantine; Coronavirus; Facebook. 


\section{Introdução}

Em dezembro de 2019 o mundo se deparou com o primeiro caso de Síndrome Respiratória Aguda Grave por Coronavírus 2 (SARS-CoV-2), uma doença que apresentou alto potencial de contágio e rapidamente se tornou emergência de saúde pública (Ornell, Schuch, Sordi, \& Kessler, 2020; Ferguson et al., 2020; Rafaell et al., 2020).

O novo coronavírus espalhou-se pelo mundo e foi categorizado pela Organização Mundial da Saúde (OMS) como uma pandemia global (Ornell, Schuch, Sordi, \& Kessler, 2020; Singh, 2020).

De acordo com dados divulgados pelo Painel de Coronavírus (2020), o primeiro caso confirmado da COVID-19 ocorreu em 26 de fevereiro de 2020 e a primeira morte ocorreu no dia 12 de março do mesmo ano. Ainda de acordo com informações divulgadas pelo Ministério da Saúde, até o dia 10 de março de 2021 o país contabilizava mais de 260.000 óbitos e mais de 11 milhões de casos confirmados (Brasil, 2021).

Com a finalidade de conter o avanço da doença, os governos adotaram 4 intervenções que combinadas tendem a trazer efeitos, são elas: distanciamento social, isolamento dos casos confirmados, fechamento de algumas instituições e quarentena familiar (Ferguson et al., 2020). O Governo chinês foi o primeiro a adotar essas medidas por ter sido o primeiro a ter contato com a doença (Xiao, 2020).

Os termos quarentena, distanciamento e isolamento social passaram a fazer parte da rotina das pessoas em todo o mundo e, mesmo apresentando significados e objetivos diferentes, aparecem como sinônimos em algumas situações (Brooks et al., 2020). Quarentena é o termo utilizado para designar a separação e limitação ao movimento de pessoas que por algum motivo foram potencialmente expostas a uma doença contagiosa, como é o caso da COVID-19 (Brooks et al., 2020). Ela é uma estratégia de saúde pública que visa reduzir os efeitos negativos associados a uma doença contagiosa. $O$ distanciamento tem como objetivo diminuir a velocidade de transmissão do vírus reduzindo a interação entre pessoas. Já o isolamento social diz respeito à medida de separação de pessoas que foram infectadas para que elas não contaminem outras pessoas, seja em casa ou no hospital, de acordo com o nível de gravidade do caso (Wilder-Smith \& Freedman, 2020).

Por outro lado, estudos apontam que a quarentena é um processo desagradável: diante da separação dos entes queridos, ausência da comunicação interpessoal, incerteza sobre estar ou não infectado, perda da liberdade e tédio, há uma eclosão de grande pressão psicológica, podendo desencadear problemas para a saúde mental, como casos de depressão e ansiedade (Xiao, 2020; Brooks et al, 2020). Atrelado a isso, as contradições e incertezas que envolvem o novo coronavírus acerca de sua transmissibilidade, período de incubação, alcance geográfico, quantitativo de infectados e taxa de mortalidade constroem espaço para um sentimento de impotência e insegurança na população (Ornell, Schuch, Sordi, \& Kessler, 2020; Shimizu, 2020).

A insuficiência das medidas de controle e a escassez de mecanismos terapêuticos eficazes também são elementos estruturantes para modificações diretas no cotidiano e na saúde mental dos indivíduos (Ornell, Schuch, Sordi, \& Kessler, 2020). Diante do sofrimento psíquico instaurado pelo afastamento social, as Redes Sociais Online (RSO) tiveram sua função intensificada enquanto facilitadoras da comunicação e compartilhamento de informações, maior aproximação entre pessoas, tornando a comunicação mais acessível (Carvalho, 2011). Nesse viés, o cenário da quarentena abriu espaço para que as RSO ganhassem ainda mais força por funcionarem como rede de apoio e externalização das crenças dos indivíduos.

A pesquisa da Tecnologia da Informação e Comunicação (TIC) destaca que, no Brasil, $69,8 \%$ da população possui acesso à internet, contribuindo para que os vínculos relacionais entre os indivíduos, através da comunicação pelas RSO, não sejam quebrados (IBGE, 2018). 
Além disso, por meio dessas manifestações pessoais, histórias são compartilhadas e formas de apoio são desencadeadas diante das incertezas acerca do vírus. Por isso, objetivou-se analisar a manifestação de crenças sobre a pandemia da COVID-19 entre usuários do Facebook no país.

\section{Metodologia}

Inicialmente foi realizado no site Facebook (http://facebook.com.br/) um levantamento no período compreendido entre maio e junho de 2020 de grupos e páginas referentes à temática investigada utilizando os descritores: COVID-19, coronavírus e quarentena na aba de pesquisa da rede social. Na oportunidade, elaborou-se os seguintes critérios de inclusão: a) ser aberto; b) abordar assuntos referentes ao eixo do estudo; c) não estar atrelado a organizações governamentais; e d) apresentar um número mínimo de $50 \mathrm{mil}$ curtidas/membros/seguidores. Após esta etapa, selecionou-se um grupo e uma página, sendo estes os mais relevantes. O grupo correspondeu a um sítio de informações compartilhadas durante a pandemia sobre o período da quarentena vivenciado no país e a página retrata uma leitura humorística das incertezas experienciadas na quarentena decorrente da pandemia.

A análise dos sítios escolhidos examinou as publicações realizadas entre 26 de fevereiro e 30 de abril de 2020, período em que as primeiras postagens relativas à COVID-19 foram publicadas e o momento de finalização das buscas. Foram selecionadas as 5 publicações mais relevantes (visualizadas, compartilhadas e/ou curtidas), sendo as mesmas compartilhadas no período temporal compreendido entre 21 de março e 16 de abril. Com a seleção das publicações foram colhidos os comentários destas, resultando em dois corpus textuais. Para analisar os dados foi utilizado software Iramuteq (Interface de R pour les Analyses Multidimensionnelles de Textes et de Questionnaires).

Para a construção do corpus, resguardou-se o anonimato de todos os indivíduos responsáveis pelos comentários. Os dados foram coletados e transcritos com o auxílio do software OpenOffice Writer (Afonso, 2010). Na sequência, realizou-se uma revisão dos dados transcritos e os produtos que não apresentavam qualquer relação com o objetivo deste estudo foram excluídos. Após a formatação e organização dos corpus, foi realizada a técnica da análise de similitude. A análise de similitude consiste em um método em que é possível identificar as coocorrências entre as palavras e seus resultados de forma a trazer indicações da conexidade do corpus textual (Camargo \& Justo, 2016).

Com a análise da conexão estabelecida entre as palavras foi possível identificar os termos com maior frequência e próximos a eles, além da visualização das coocorrências no corpus. O tamanho de cada palavra indica sua importância no corpus e a espessura das linhas que ligam cada termo indicam o grau de associação entre eles, de modo que quanto mais espessa a linha, maior será a associação. Este tipo de análise permite também a identificação de partes comuns e de especificidades entre os vocábulos a partir do agrupamentos observado na imagem (Camargo \& Justo, 2016).

\section{Resultados}

Inicialmente, realizou-se um levantamento no Facebook considerando grupos e páginas abertos que tratassem de conteúdos referentes às estratégias de compartilhamento de crenças entre os brasileiros diante das incertezas trazidas pela pandemia da COVID-19 (Tabela 1). 
Tabela 1. Sítios selecionados para a composição da amostra.

\begin{tabular}{cccc}
\hline Característica & Tipologia & $\begin{array}{c}\text { Seguidores/ } \\
\text { membros }\end{array}$ & $\begin{array}{c}\text { Período das } \\
\text { publicações analisadas }\end{array}$ \\
\hline Publicações humorísticas & Página & 136.425 mil & $\begin{array}{c}19 \text { de março de } 2020 \text { até } \\
30 \text { de abril }\end{array}$ \\
Publicações informativas & Grupo & 51 mil & $\begin{array}{c}26 \text { de fevereiro de } 2020 \\
\text { até } 30 \text { de abril }\end{array}$ \\
\hline
\end{tabular}

As 5 publicações extraídas do sítio humorístico abordavam os seguintes aspectos: 1) Problemas relacionados à assistir televisão durante a pandemia; 2) Técnicas para aprender a tocar um instrumento musical na pandemia; 3) Discussão de pessoas embriagadas que não se tocam por conta do distanciamento social; 4) Pescaria durante a quarentena e 5) Pedido de ajuda durante a pandemia. As 5 publicações do sítio de cunho informativo são: 1) Relato de experiência na quarentena; 2) Discussão sobre o recebimento de visitas em casa nesse período; 3) Idoso que faleceu por COVID-19; 4) Relato de uma mãe sobre a preocupação com a saúde de seu filho e 5) Posicionamento do presidente do Brasil a favor da reabertura de escolas no país.

Tabela 2. Caracterização das postagens.

\begin{tabular}{lccc}
\hline \multicolumn{1}{c}{ Categoria } & Total de curtidas & Total de comentários & Data de publicação \\
\hline Humor & $11 \mathrm{mil}$ & 806 & 23 de março \\
Humor & $7,1 \mathrm{mil}$ & $1,3 \mathrm{mil}$ & 21 de março \\
Humor & $6,4 \mathrm{mil}$ & 706 & 2 de abril \\
Humor & $5,7 \mathrm{mil}$ & $13 \mathrm{mil}$ & 21 de março \\
Humor & $4,6 \mathrm{mil}$ & $1,1 \mathrm{mil}$ & 31 de março \\
Informação & $1,7 \mathrm{mil}$ & 286 & 26 de março \\
Informação & $1,2 \mathrm{mil}$ & 201 & 1 de abril \\
Informação & $1 \mathrm{mil}$ & 468 & 27 de março \\
Informação & $1 \mathrm{mil}$ & 101 & 10 de abril \\
Informação & 976 & $1,366 \mathrm{mil}$ & 16 de abril \\
\hline
\end{tabular}

Foi possível observar que o número de curtidas e comentários demonstram uma maior participação dos usuários das RSO em conteúdos voltados para o humor. As informações compartilhadas entre os indivíduos também ganham força nesse período, principalmente por se tratar de um momento de incertezas a respeito dos sintomas e transmissão do vírus, fator que fica evidente nos comentários de relato de pessoas que enfrentaram a doença ou de conhecidos.

Em seguida, realizou-se uma análise de similitude com o conteúdo das 5 postagens referentes à página selecionada. A árvore foi disposta através da interface dos resultados da análise do corpus "Humor" (Figura 1). A identificação das simultaneidades e conexão entre os termos pai, amigo e quarentena, são aspectos que possibilitam a compreensão dos fatores associados às crenças referentes ao enfrentamento da COVID-19. 


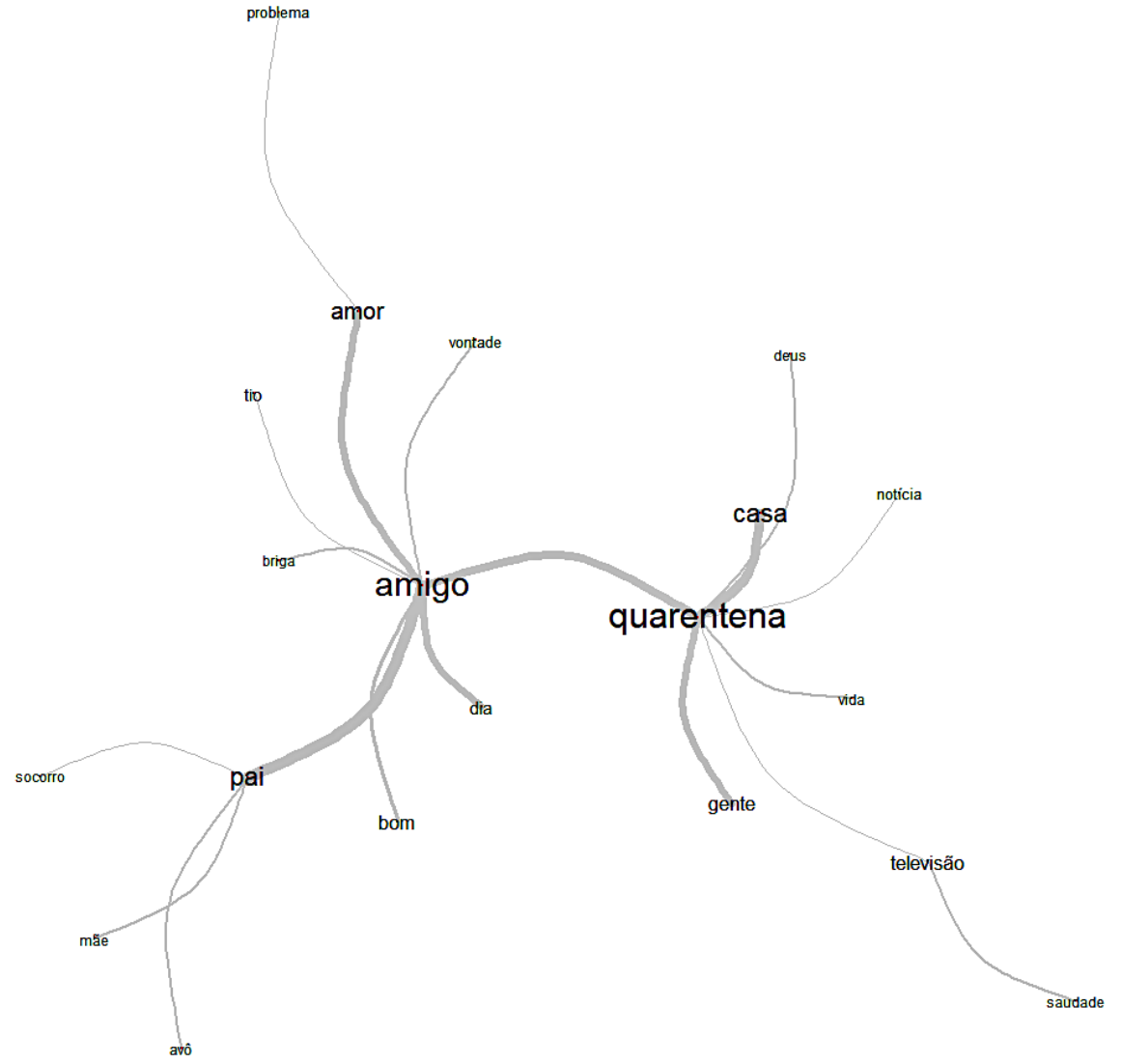

Fig. 1. Resultado da análise de similitude do corpus Humor.

De acordo com a árvore de coocorrência, os resultados apontam para a existência de dois eixos nas estratégias de enfrentamento utilizadas pelos usuários da página de humor analisada, um eixo de estratégias positivas, e o segundo eixo referente a estratégias negativas. A saber: 1) Rede de apoio social e afetiva e 2) Compartilhamento de aspectos negativos da quarentena.

O conjunto de estratégias de enfrentamento positivas referentes à construção de uma rede de apoio é utilizado enquanto uma ferramenta de proteção. A palavra central deste eixo é "amigo", vocábulo este que se liga ao termo secundário "pai" que se apresenta como uma importante palavra que desencadeia outras conexões. A presença dos vocábulos "tio", "amor", "mãe" e "avô" sustentam essa afirmativa, os seguintes trechos dos comentários contribuem para essa interpretação: "Olha que ótima saída pra nós que não temos o que fazer na quarentena amor, sogra e amigo", "Fala para o nosso amigo fazer isso durante a quarentena".

Já o segundo eixo, que se relaciona com o compartilhamento de aspectos negativos da quarentena é apreendido como uma estratégia negativa de enfrentamento ao contexto de incertezas. O vocábulo central deste eixo é "quarentena", que apresenta conexões com palavras secundárias como "casa" e "gente", outras palavras ocupam destaque nesse eixo, como por exemplo "televisão" e "notícia". É possível observar em: "Uma coisa é certa, o isolamento social e a quarentena são inúteis porque quando chega o momento de você morrer, ninguém sabe a hora ou o local, pode ser fora ou dentro de casa". 
A árvore do corpus "Informação" (Figura 2), por sua vez, apresenta os elementos centrais: "deus", "filho" e "pessoa". Assim, esse método permite a identificação da estrutura do corpus auxiliando na associação das crenças que emergem a partir das informações e discussões sobre a COVID-19 entre usuários do Facebook.

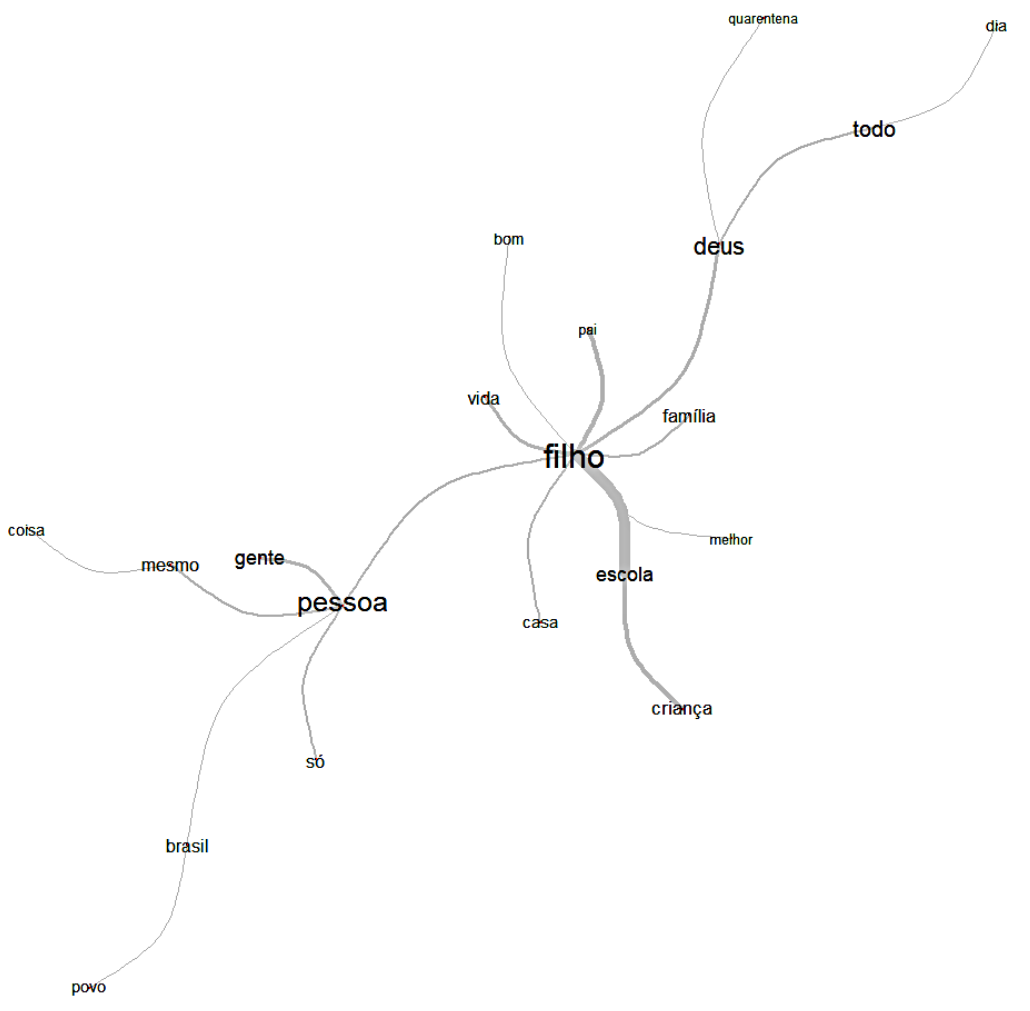

Fig. 2. Resultado da análise de similitude do corpus "Informação".

Diante da árvore de coocorrências, nota-se que os resultados demonstram que uma das formas de dialogar dos usuários do grupo analisado remete ao compartilhamento de notícias e informações com foco no: 1) Auxílio religioso; 2) Cuidado com a família e 3) Condição física e mental das pessoas.

O auxílio religioso se relaciona ao apego à figura religiosa como forma de lidar diariamente com as diversas notícias de casos e óbitos ocasionados pela COVID-19. Assim, as palavras em destaque na árvore e seus segmentos de texto refletem isso: "Vamos nos prevenir e fazer o certo, tá nas mãos de Deus o nosso destino mas se não nos cuidarmos aí fica difícil mais ainda, eu estou aproveitando para refletir, vejo que se ficarmos vendo tv piraremos mais ainda, estou seguindo as orientações devidas e agradecendo a Deus por estar equilibrada pois sei que abala o emocional com toda certeza...".

O cuidado com a família encontrado nos termos "filho", "família" e "pai" apresenta-se como uma forma de buscar a segurança ("vida" e "casa") e encontrar o controle diante da situação de calamidade pública e das notícias de retorno às atividades cotidianas como o retorno de "crianças" à escola, mesmo diante dos casos de crianças que contraíram o Vírus: "...vamos lutar contra a volta das crianças pra escola e a quem não quer arriscar a vida no trabalho temos que cada um fazer a sua parte com o povo, ele não vai perder, e nós orar e colocar Deus na frente e nos cuidar." 
Diante do contexto da palavra central "pessoa" verifica-se a preocupação e o cuidado com o estado físico e mental da população: "Torça para não ter conhecidos ou familiares fazendo parte das estatísticas que estão por vir pois tem pessoas que mesmo não fazendo parte do grupo de risco foram infectados e morreram e você vai ser um número para o governo do Brasil".

\section{Discussão}

O excesso de informações enganosas, o medo da doença e de suas consequências pode causar um efeito psicológico considerável (Đogaš et. al, 2020). Esses impactos psicológicos podem fazer com que essas consequências sejam duradouras e substanciais (Brooks et. al, 2020). Dentre as reações mais frequentes se observa a angústia emocional, comportamentos inadequados e respostas defensivas como a ansiedade, a frustração, a depressão e os comportamentos de evitação (Talevi, 2020). Estudos apontam a existência de três estratégias de enfrentamento a situações estressantes: 1) resolução de problemas, 2) evitação de problemas e 3) respostas emocionais (Amici, 2020).

A utilização das tecnologias e a divulgação rápida de informações intensifica o pânico e a ansiedade da população (Dong \& Zheng, 2020). Entretanto, as notícias podem ser apresentadas através de duas formas distintas: informação e humor (Rosenberg, Syed \& Rezaie, 2020). As informações que são compartilhadas não devem ser apreendidas considerando sua total veracidade, nem toda a população recebe informações adequadas sobre os problemas de saúde que as afetam, e essa desinformação pode ter um impacto severo na sensação de segurança das pessoas, principalmente no contexto pandêmico, gerando agravos à saúde mental (Henriques, 2018; Laato, Islam, Islam \& Whelan, 2020).

O empenho em práticas religiosas possibilita uma percepção de controle sobre a situação, pois auxilia na ressignificação do evento problemático e estressante (Kowalczyk et. al, 2020). Com o poder de difusão de informações quase que instantâneo da internet, além das informações provenientes de fontes confiáveis, as notícias falsas também rapidamente se espalham (Galhardi, Freire, Minayo \& Fagundes, 2020). Abre-se espaço para que informações sem embasamento científico disseminem inverdades e acarretem um aumento dos casos da doença (Avaaz, 2020). Esse excesso de informação sobre um mesmo tema tem sido denominado pela literatura como Infodemia (OPAS, 2020). Nesse viés, causa uma desorientação nas pessoas, prejudicando a percepção sobre a confiabilidade das fontes e conteúdos, e propiciando a aceitação como verdade daquilo que convém às suas crenças e valores (Henriques, 2020).

Apesar dessa desorganização social forjada pela pandemia e todos os aspectos negativos a ela atrelados, há também influências positivas exercidas pelas redes sociais. $O$ compartilhamento de apoio através de informações positivas é um elemento importante em um esforço para quebrar a cadeia de desesperança produzida pelo vírus (Sulistyaningtyas, Jaelani \& Suryani, 2020). Na perspectiva positiva do compartilhamento de informações, o humor se apresenta como uma estratégia de combate ao isolamento social e de promoção ao bem-estar psicológico durante esse cenário pandêmico nas RSO. Essa categoria construída é denominada de humor benigno podendo ser tomada como um mecanismo de enfrentamento que pode auxiliar no aumento do companheirismo (Chiodo, Broughton \& Michalski, 2020).

A utilização do humor em um contexto pandêmico possibilita a ressignificação de uma rede de apoio, sendo os laços presenciais transpostos para o meio digital (Amici, 2020). A mobilização da rede social é um fator de proteção à exposição de eventos estressantes, uma vez que o apoio dos indivíduos pode auxiliar na redução das consequências negativas do estresse na saúde (Costa \& Leite, 2009). Apesar do humor geralmente ser generalizado e classificado como facilitador físico e mental, ele é erroneamente colocado em uma posição de somente conceder benefícios, também existe a faceta prejudicial de sua utilização (Chiodo, Broughton \& Michalski, 2020; Kuiper, Grimshaw, Leite \& Kirsh, 2004). 
Seus efeitos negativos são decorrentes de um humor autodestrutivo, estando associado com maiores níveis de distúrbios na saúde mental, podendo aumentar o estresse, a ansiedade e diminuir a autoestima (Kuiper, Grimshaw, Leite \& Kirsh, 2004).

\section{Conclusões}

A análise das manifestações de crenças compartilhadas entre usuários do Facebook diante da pandemia da COVID-19 no país nos possibilitou traçar um olhar à luz do comportamento social em um período de crise sanitária de proporção global. Apesar da humanidade ter enfrentado outros árduos e dolorosos processos pandêmicos, ainda não havia uma ferramenta de comunicação social capaz de disseminar uma informação de forma instantânea como possibilitada hoje por meio da rede de internet.

No contexto da pandemia da COVID-19, as premissas de afastamento social e demais medidas preventivas, tiveram o Facebook como um importante canal de comunicação, compartilhamento, engajamento, apoio social e estratégias de enfrentamento baseadas em dois eixos aqui analisados: humor e informação frente à COVID-19 na modalidade virtual. Porém, faz-se importante destacar o quanto é desafiador lidar com um conjunto de informações e condições adversas resultantes das incertezas dos impactos decorrentes da pandemia. Algo que não pode ser esquecido é o papel dos impactos biopsicossociais sofridos pela população que possivelmente desencadearam a necessidade de novas estratégias, possivelmente em uma sociedade com maiores incidências psicopatológicas.

É preciso ponderar alguns aspectos que podem ser limitantes das conclusões levantadas neste estudo. Ao investigar apenas uma rede social, é necessário reconhecer a possibilidade de haver um viés disparado pela utilização de robôs e a disseminação de fake news, sendo interessante a ampliação das análises para outras redes sociais e canais de comunicação, a exemplo do Instagram, Twitter ou WhatsApp. Importante também considerar a análise das crenças em outras fases da pandemia, em especial nos períodos em que os governos estaduais e municipais assumem distintamente a decisão de fechamento de bares, restaurantes, escolas e implantam as atividades de estudo e trabalho remoto.

\section{Referências}

Afonso, P. (2020). The Impact of the COVID-19 Pandemic on Mental Health. Acta Médica Portuguesa, 33(5), 356-357.

Amici, P. (2020). Humor in the age of Covid-19 Lockdown: An explorative qualitative study. Psychiatria Danubina, 32, S15-S20.

Avaaz. (2020). O Brasil está sofrendo uma infodemia de Covid-19. In Avaaz. Disponível em: https://avaazimages.avaaz.org/brasil_infodemia_coronavirus.pdf

Brasil. (2021). COVID19 Painel Coronavírus. Disponível em: https://covid.saude.gov.br

Brooks, S. K., Webster, R. K., Smith, L. E., Woodland, L., S Wessely, S., Greenberg, N., \& Rubin, G. J. (2020). The psychological impact of quarantine and how to reduce it: rapid review of the evidence. The Lancet, 395, 912-20.

Camargo, B. V.; Justo, A. M. (2016) Tutorial para uso do software IRAMUTEQ. Laboratório de Psicologia Social da Comunicação e Cognição: Universidade Federal de Santa Catarina.

Carvalho, J. H. D. (2011). A publicidade nas redes sociais e a Geração Y: A emergência de novas formas de comunicação publicitária. Revista Negócios Em Projeção, 2(2), 91-105.

Chiodo, C. P., Broughton, K. K., \& Michalski, M. P. (2020). Caution: Wit and Humor During the COVID-19 Pandemic. Foot and Ankle International, 41(6), 763-764. 
Costa, P., \& Leite, R. C. B. O. (2009). Estratégias de enfrentamento utilizadas pelos pacientes oncológicos submetidos a cirurgias mutiladoras. Revista Brasileira de Cancerologia, 55(4), 355-364.

Đogaš, Z., Kalcina, L. L., Dodig, I. P., Demirović, S., Madirazza, K., Valić, M., \& Pecotić, R. (2020). The effect of COVID-19 lockdown on lifestyle and mood in Croatian general population: $A$ cross-sectional study. Croatian Medical Journal, 61(4), 309-318.

Dong, M., \& Zheng, J. (2020). Letter to the editor: Headline stress disorder caused by Netnews during the outbreak of COVID-19. Health Expectations, 23(2), 259-260.

Ferguson, N. M., Laydon, D., Nedjati-Gilani, G., Imai, N., Kylie, A., Baguelin, M., Bhatia, S., Boonyasiri, A., Cucunubá, Z., Gina, C.-D., Amy, D., Ilaria, D., Han, F., Katy, G., Will, G., Arran, H., Wes, H., Okell, L. C., Sabine, van E., ... Azra C, G. (2020). Impact of nonpharmaceutical interventions (NPIs) to reduce COVID-19 mortality and healthcare demand. Imperial College COVID-19 Response Team, March.

Galhardi, C. P., Freire, N. P., Minayo, M. C. D. S., \& Fagundes, M. C. M. (2020). Fato ou Fake? Uma análise da desinformação frente à pandemia da Covid-19 no Brasil. Ciência \& Saúde Coletiva, 25, 4201-4210.

Henriques, C. M. P. (2018). A dupla epidemia: febre amarela e desinformação TT - Two epidemics: the yellow fever and a lack of information TT - La dupla epidemia: la fiebre amarilla y la falta de información. RECIIS (Online), 12(1), 1-5.

Henriques, C. M. P., \& Vasconcelos, W. (2020). Crises dentro da crise: Respostas, incertezas e desencontros no combate a pandemia da Covid-19 no Brasil. Estudos Avancados, 34(99) $25-44$

IBGE. (2018). Pesquisa Nacional por Amostra de Domicílios. In IBGE (p. 132). https://agenciadenoticias.ibge.gov.br/media/com_mediaibge/arquivos/81c9b2749a7b8e5b67f 9a7361f839a3d.pdf

Kowalczyk, O., Roszkowski, K., Montane, X., Pawliszak, W., Tylkowski, B., \& Bajek, A. (2020). Religion and Faith Perception in a Pandemic of COVID-19. Journal of Religion and Health, 59(6), 2671-2677.

Kuiper, N. A., Grimshaw, M., Leite, C., \& Kirsh, G. (2004). Humor is not always the best medicine: Specific components of sense of humor and psychological well-being. Humor: International Journal of Humor Research, 17(1-2), 135-168.

Laato, S., Islam, A. K. M. N., Islam, M. N., \& Whelan, E. (2020). What drives unverified information sharing and cyberchondria during the COVID-19 pandemic? European Journal of Information Systems, 29(3), 288-305.

Li, L., Zhang, Q., Wang, X., Zhang, J., Wang, T., Gao, T. L., Duan, W., Tsoi, K. K. F., \& Wang, F. Y. (2020). Characterizing the Propagation of Situational Information in Social Media during COVID-19 Epidemic: A Case Study on Weibo. IEEE Transactions on Computational Social Systems, 7(2), 556-562.

OPAS. Organização Pan-Americana da Saúde (2020). Repositório Institucional para Troca de Informações - Iris. Fichas Informativas COVID-19: entenda a infodemia e a desinformação na luta contra a COVID-19 [Internet]. Brasília: Organização Pan-Americana da Saúde. Disponível em: https://iris.paho.org/handle/10665.2/52054?locale-attribute=pt

Ornell, F., Schuch, J. B., Sordi, A. O., \& Kessler, F. H. P. (2020). "Pandemic fear" and COVID-19: mental health burden and strategies. Brazilian Journal of Psychiatry, 42(3), 232-235.

Rafaell, R. de M. R., Neto, M., Carvalho, M. M. B. de, David, H. M. S. L., Acioli, S., \& Faria, M. G. de A. (2020). Epidemiologia, políticas públicas e pandemia de Covid-19: o que esperar no Brasil? Rev. Enferm. UERJ, 2-7.

Rosenberg, H., Syed, S., \& Rezaie, S. (2020). The Twitter pandemic: The critical role of Twitter in the dissemination of medical information and misinformation during the COVID-19 pandemic. Canadian Journal of Emergency Medicine, 22(4), 418-421.

Shimizu, K. (2020). 2019-nCoV, fake news, and racism. The Lancet, 395(10225), 685-686. 
Singh, R. K., Rani, M., Bhagavathula, A. S., Sah, R., Rodriguez-Morales, A. J., Kalita, H., Nanda, C., Sharma, S., Sharma, Y. D., Rabaan, A. A., Rahmani, J., \& Kumar, P. (2020). Prediction of the COVID-19 pandemic for the top 15 affected countries: Advanced autoregressive integrated moving average (ARIMA) model. JMIR Public Health and Surveillance, 6(2), 1-10.

Sulistyaningtyas, T., Jaelani, J., \& Suryani, Y. (2020). Power of Knowledge and Community Social Class above Covid-19 Pandemic Information on Social Media. Jurnal Komunikasi Ikatan Sarjana Komunikasi Indonesia, 5(1), 52-62.

Talevi, D., Socci, V., Carai, M., Carnaghi, G., Faleri, S., Trebbi, E., Bernardo, A. D. I., Capelli, F., \& Pacitti, F. (2020). Mental health outcomes of the CoViD-19 pandemic Gli esiti di salute mentale della pandemia di CoViD-19. Riv Psichiatr, 55(3), 137-144.

Wilder-Smith, A., \& Freedman, D. O. (2020). Isolation, quarantine, social distancing and community containment: Pivotal role for old-style public health measures in the novel coronavirus (2019nCoV) outbreak. Journal of Travel Medicine, 27(2), 1-4.

Xiao, C. (2020). A novel approach of consultation on 2019 novel coronavirus (COVID-19)-related psychological and mental problems: Structured letter therapy. Psychiatry Investigation, 17(2), 175-176. 